\title{
Common and Domain-specific Metamodel Elements for Problem Description in Simulation Problems
}

\author{
Patrizia Ribino ${ }^{\ddagger}$, Valeria Seidita ${ }^{\S \ddagger}$, Carmelo Lodato ${ }^{\ddagger}$, Salvatore Lopes ${ }^{\ddagger}$ and Massimo Cossentino $o^{\ddagger}$ \\ ${ }^{\ddagger}$ Istituto di Reti e Calcolo ad Alte Prestazioni \\ Consiglio Nazionale delle Ricerche \\ Palermo, Italy \\ Email: \{ribino,c.lodato,lopes,cossentino\}@pa.icar.cnr.it \\ $\S$ Dip. di Ingegneria Chimica Gestionale Informatica Meccanica, University of Palermo \\ Palermo, Italy \\ Email: \{valeria.seidita\}@unipa.it
}

\begin{abstract}
It is well known that the multi-agent system paradigm is well suited for modelling and developing simulations of complex systems belonging to several application domains. Simulation study aims at developing simulation models useful for representing, studying and analyzing entities and their behavior in a system according to specific purposes. With our work we are trying to understand what are the right elements to be considered and included in the description of a simulation problem. In order to root our resulting metamodel in the state of the art of multi-agent simulations we started from the study of twelve papers dealing with four different application domains: Crowd Dynamics, Traffic and Transportation, Electricity Power Engineering and Supply Chain and Logistic. From this study we obtained a metamodel that may be used by an analyst as a guideline and concept repository for facing a new system design. The metamodel is the result of a well defined approach that is described together with the obtained results consisting in one core metamodel containing elements that are common to all the four application domains and some domain extension contents. These latter contain the elements that are specific of each of the studied domains and are not present in the others.
\end{abstract}

\section{INTRODUCTION}

$\mathbf{M}$ ULTIAGENT system paradigm is well suited for modeling and developing simulations of complex systems belonging to several application domains. As Klügl says in [1], multiagent simulation is considered a killer application of agent-based technology. But an engineering approach for developing simulation models is still lacking and several issues are still open. Moreover the author says " a categorization of models with respect to relevant design concepts would be a good starting point advancing the knowledge on what is relevant for multiagent simulations "[1]. This future research is suggested to address the challenge about: which design concepts are relevant for which type of model? Due to the wide variety of domains in which multiagent simulations are employed, our question is: which concepts are relevant for which type of agent based simulation problem? Answering to this question, in our opinion, helps to find a solution to the proposed challenge.

The main aim of our research is to develop a complete methodological approach for conducting simulation studies. Our approach will be composed of: (i) the phase devoting to describe the features of the simulation problem, (ii) the phase for verifying if the simulation model adheres to the real system and then (iii) the phase for defining experiments and implementing them in the simulation framework. The development of a specific MAS resides inside the whole simulation methodology.

In this paper we focus on the first part of our project, the one related to the first activities of a simulation study. Simulation study aims at developing simulation models useful for representing, studying and analyzing entities and their behavior in a system following specific purposes. Activities in simulation studies are mainly performed by the analyst and the model designer that have to create a model of the world starting from the description of the problem. But what are the right tools for them to build the simulation model that best fits their needs? And above all, agent simulation is used in several application domain presenting very different features that affect the creation of simulation models, so how may the model designer identify the elements helping him to construct the simulation model?

This starting point is very demanding, several authors in literature agree on that and propose different solutions. In this work we propose a preliminary work for solving this problem by focusing on the problem statement, our aim is to create a metamodel of all the elements and their relationships that we have to find in the problem description of a simulation study. As we said, several different simulation studies greatly differ for the application domain they refer to, so in order to be as much general as possible in the creation of the metamodel, we analyzed and studied papers by four different application domains (Crowd Dynamics, Traffic and Transportation, Electricity Power Engineering and Supply Chain and Logistic) in order to retrieve common and specific elements.

In other words, the contribution of this paper is in the identification of what elements are commonly used for describing some simulation problems and, moreover, what elements are used in specific application domains. From this study we obtained a metamodel that may be used by an analyst as a guideline and concept repository while describing a new problem. We do not claim that all situations may be faced 
(or have to be faced) with the proposed metamodel but we think that if the elements we list in that have been frequently used in papers dealing with similar problems, it is likely that such elements may be useful again in the same context or in a similar one.

The rest of the paper is organized as follows: in section II we detail the motivations for our work against the related works in literature, in section III we show the process for bundling the metamodel and then in sections IV and V some discussions and conclusions are drawn.

\section{Motivation AND Related Works}

Simulation is the discipline for designing a model of actual or physical systems. It abstractly represents a real system involving the elaboration of models where system behavior is reproduced following a set of hypothesis used to define different scenarios. Multiagent simulation focuses on the study and description of distributed behavior in a dynamic context [2] and consists in identifying simulation models where entities (such as agents), their behaviors, their interactions among themselves or with the environment in which they are situated, are described. Hence simulation studies prescribe to work with models of the problem and not with the problem itself.

Simulation results are used in place of experimentation over the actual or real system. If the model were not the closest approximation of the actual system then it might lead to erroneous considerations, faults and costly decisions.

The question we want to answer is: which are the activities to made in order to build adequate simulation models?

In [3] and [4] the life cycle of a simulation study is illustrated, it prescribes ten phases organized in a quite iterative way. Briefly, the first step is communicating the problem and formulating it, then defining the system objectives, creating the conceptual model and finally designing experiments. Defining and setting the objectives of the study is a very important step that may lead to the identification and the definition of the right simulation model to use for investigating a specific problem and analyzing its results.

In [5] the author investigates how to build a valid and credible simulation model. He does not describe a real and complete methodological approach, rather he presents a set of techniques for building a valid model. Law's approach starts with the identification of a set of steps to do for formulating the problem, these steps sound like advises more then techniques, indeed he say "Problem of interest is stated by the decisionmaker. It may not be stated precisely or in quantitative terms. An iterative process is often necessary" and still "a kickoff meeting is necessary for discussing the overall objectives of the study, the scope of the model, the performance measures, etc.". Nothing is said, in Balci's, Nance's and Law's works, about how to perform these activities in order to retrieve the most useful elements for creating simulation models starting from problem description.

It is clear that the description of the problem, or of the domain under study, greatly affects the production of simulation models. The risk of influencing the result of simulation study is high. The model developer and then the decision maker have to be sure to work with problem domain description where useful elements are described. From a literature review and above all from [6] we can highlight the following elements: entities of the domain exposing behavior, the objective of the simulation, the parameters to be tuned for evaluating results, the interactions among entities, possible rules or constraints for interacting, resources and services from which entities take data for implementing their behavior.

Balci and Nance in [7] presented an high level procedure for guiding the analyst during problem formulation; he identified the need of formulating the problem in objective terms and distinguished between problems requiring prescriptive or descriptive solutions. The procedure he proposed is very detailed and is followed by a formulated problem verification activity and the measurement of the formulated problem. The overall approach may be resumed in fifteen very detailed tasks that, we may say, influenced all the successive work in problem domain description and from which the previous said elements may also be abstracted.

In the agent oriented context Garro et al. [8] propose a methodology for guiding domain experts from analysis to modeling. Our approach mainly focuses on the problem formulation and, starting from the same considerations of Balci and Nance, does not "still" propose detailed guidelines for problem statement description but instead provides the set of elements and relationships among them, a metamodel, that we abstracted from several works presented in literature. This metamodel is the base for obtaining the sequence of activities to do for building the most correct problem statement for a specific problem.

Today, it is quite usual that existing software engineering design methodologies start with the assumption that the Problem Statement document is already available, it is usually presented in the form of a text document, sometimes delivered using techniques such as interviews or ethnographic approaches [9] as often as not it is presented as an unstructured document containing a free description of the problem.

Our aim is to pose the base for creating guidelines for writing problem statements as a useful input for an agentbased simulation methodology and we want to answer to the following questions:

- How the problem statement has to be done?

- $\quad$ Which are the essential elements it has to present?

- How to describe the specific problem that should be solved by the model in the simulation study?

First of all, we considered that if the Problem Statement has to serve as a basis for obtaining a simulation model it has to contain and make explicit elements such as the entities involved in the real system, their behavior and interaction with the environment but also elements representing the objectives of the simulation and a fair set of parameters settings and measures.

This is very different from a common problem statement, for instance from the one devoted to illustrate elements useful for obtaining an object model (of a object-oriented methodology). 


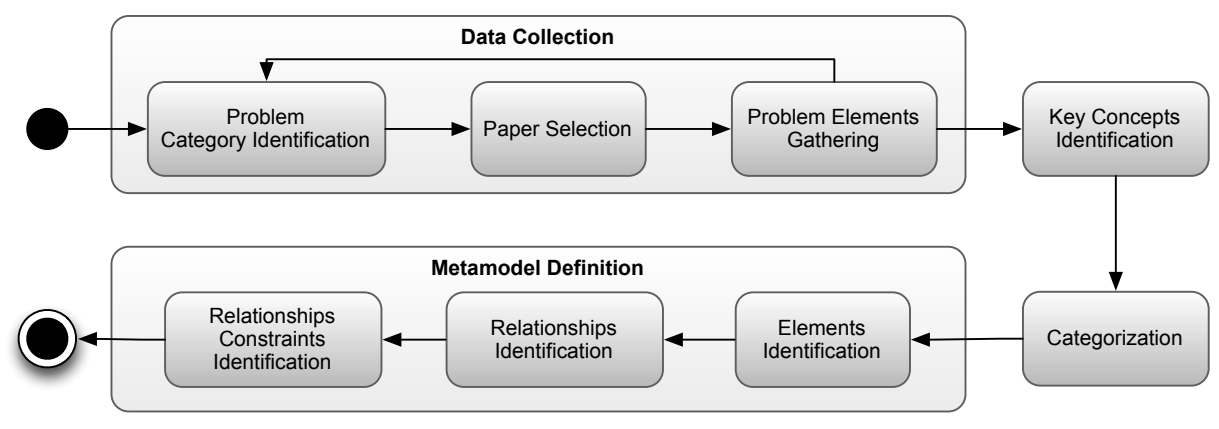

Fig. 1. Metamodel Building Process

In what does a simulation study differ from an agent based methodology? May differences be reported on the structure (or on how to build) of a problem statement? In order to answer to these questions we decided to explore and analyze the research products of several fields in which agent-based simulation is used in order to retrieve the common elements in use and to create a metamodel for supporting the creation of problem statements for agent-based simulation systems.

We analyzed literature from the fields of Financial Market, Urban Development, Traffic and Transportation, Crowd Dynamics and Logistic and Supply Chain Management in order to identify the shared elements in all these categories of problems and to conduct a generalization process that is detailed in the following section.

The reason for which we create a metamodel for representing abstract simulation study elements lies in the deep experience we have in creating and documenting design methodologies [10], [11], [12], [13]. Indeed, in the PRoDe approach [14], elements and relationships among them are used for representing dependencies among elements and establishing the right sequence of tasks/activities to do for reaching a design result. This is what we mean to do with the problem domain, once having determined the elements and their relationships that have to be present in a problem statement we might be able to determine the set of activities for instantiating them. This results, in the context of our complete building-in- progress methodology, in a set of guidelines to be used for writing the most useful problem statement for a specific need.

\section{Metamodel DEFINITION}

A critical point for a simulation study is to accurately formulate the problem to be addressed in order to obtain a high-quality simulation model. Problem statement is the first activity by which the addressed problem is translated into a well formulated one that is sufficiently detailed for allowing specific modeling activities. Several studies have highlighted that agent-based simulations seem to be more suited in the field of socio-economic systems, which covers a wide range of problem domains. These domains share some common concepts but differ from some others. Hence, for answering the question "which concepts are relevant for specific types of agent based simulation problems?", we are leading a systematic analysis of several scientific papers in order to determine: (i) what are the domains of simulation problems commonly faced with an agent-based approach; (ii) what is the set of shared concepts useful for describing such problems and (iii) what is the set of concepts that differ among domains of agent based simulation problems.

As we previously said, we are working on the development of a methodology that covers the entire life cycle of a simulation study starting from the problem statement activity. In this paper we propose a preparatory study whose aim is building a metamodel that may be applicable in different agent based simulation studies for supporting the problem statement creation activity.

In the following we show the process we performed for building such metamodel and its preliminary results.

\section{A. Metamodel Building Process}

In order to build the metamodel for agent based simulation problems, we followed the process shown in Fig.1. The first three steps allow us to collect data and information that will be the starting point for our analysis. Then, a key concepts identification, followed by a categorization activity, enables us to make some reasoning useful for determining the metamodel. In the following we show the results of these steps.

a) Data Collection Phase: Firstly, we performed a literature review for identifying problem categories addressed by means of agent based simulation approaches. The preliminary results of this research highlighted that agent based simulations are applied to solve problems in the following domains (and not only ${ }^{1}$ ): (i) Financial Markets [15], [16] where simulations are used for studying the behavior of individual investors, the dynamics of markets, trading mechanism and so on; (ii) Urban Development [17], [18] that studies models for urban planning, city dynamics, individual residential behaviors; (iii) Traffic and Transportation [19], [20], [21] dealing with simulation models for transportation planning, design and operations; (iv) Crowd Dynamics [22], [23], [24] that studies the behaviors of individuals, groups in several critical scenarios often with the

\footnotetext{
${ }^{1}$ This research is not intended to be exhaustive. The results we present are only preliminary. We are doing further researches in order to obtain more accurate results.
} 
aim of buildings design; (v) Social Networks that studies the evolution and dynamics of networks [25], [26]; (vi) Logistics and Supply Chain Management that studies processes inside different nodes of supply chain or the whole supply chain in order to find the best organizational structure for collaborative companies working together [27], [28], [29]; (vii) Electric Power Engineering [30], [31], [32] deals with the generation, transmission, distribution and utilization of electric power as well as the electrical devices connected to such systems.

The second step in the Data Collection phase was papers selection for each of the previously identified categories. The selection was based on two criteria: details of the simulation problem description and its relevance/impact of the paper. Finally, we analytically gathered elements from each paper. Such elements were opportunely ordered according to their semantic and functional similarity.

So far, we have examined only four problem domains: Traffic and Transportation [19], [20], [21], Crowd Dynamics [22], [23], [24], Electric Power Engineering [30], [31], [32], Logistics and Supply Chain Management [27], [28], [29]. In this preliminary work we selected 12 papers, three for each domain.

The results of this phase are sets of domain dependent concepts. All the common concepts of each domain have been grouped by analyzing the text describing each domain in the papers. Each element has been identified firstly looking at its explicit presence in sentences, the same was made for relationships, and secondly analyzing the whole domain description text in order to find unexpressed implicit knowledge.

b) Key Concepts Identification and Categorization: The key concepts identification (see Fig.1) is the conceptual activity we performed for processing sets of domain dependent concepts coming from the previous stage. Our aim was to infer a set of "higher level" abstraction categories that can be more widely applicable to generic agent based simulation problems (see Fig.2). For instance, in the domains we studied, we found concepts like room, road and city that may be represented, at an higher level, by the "spatial position" concept.

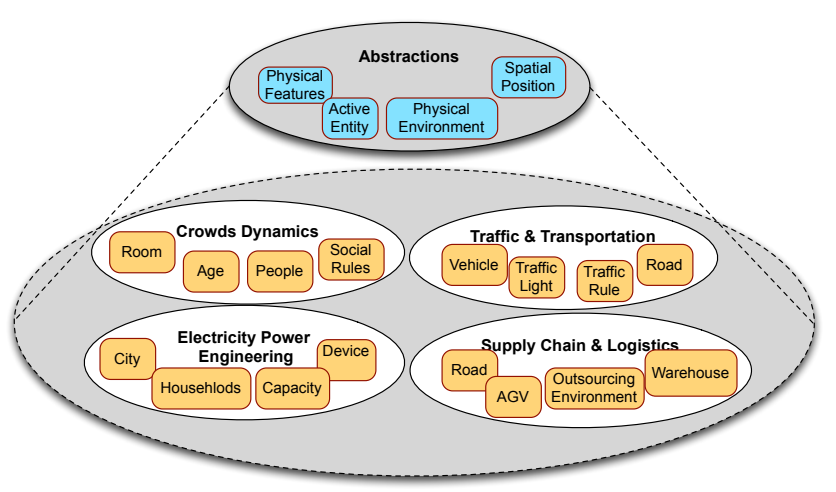

Fig. 2. Identification of higher level abstraction categories for the problem description metamodel.
The results of this activity are listed below:

1) A problem statement of an agent based simulation study describes active entities. For representing such entities, each domain uses concepts such as: (i) people, persons, pedestrian, individuals, human beings in the field of crowd dynamics; (ii) cars, drivers, buses, vehicles, people, AGVs, trucks etc... in the domain of traffic and transportation; (iii) electric cars, devices, households, electricity sellers, buyers, players etc... in the domain of power engineering; (iv) companies, suppliers, production, customers, resources,organization etc... in the domain of logistic and supply chain management.

2) Active entities may be located in a physical space. Their local position could either be a geographic position or a particular place (for example a room, a corridor, a road, in a station and so on);

3) Active entities are described by means of features and behaviors. The kinds of features we discovered in our domains are: physical, psychological and technical features. To give some examples in the domain of Crowd Dynamics a problem statement may contain sentences such as: "Agents in a hurry will not respect others' personal space ... More polite agents will respect lines and wait for others to move first..." [23]; "Agents are given different psychological (e.g., impatience, panic, personality attributes) and physiological traits" [23]; "Human individuals are different from each other by age, body dimension, mobility and personality"[33]. Examples in Power Engineering are: "The market operator manages the pool using a market-clearing tool to set market price and a set of accepted selling and buying bids for every negotiation period" [30]. "Room air conditioning Schedule time on: $15 \mathrm{~min}$ Schedule time off: 35 min, Power: $221 \mathrm{~W}$... The simulated vehicles are able to move to and between different cities and recharge from time to time" [31]. In the domain of Traffic and Transportation, typical features are described with sentences such as "Each driver or pedestrian is assigned an agent's profile that includes height, width, velocity steering ..." [21]. In the Logistic and Supply Chain domain we may find sentences such as "...forest is harvested by small-size entrepreneurs responsible for felling trees, for cross cutting them into appropriate length logs, and for hauling then to roadside" [34].

4) The problem statement includes a description of a real world portion (physical or abstract). The represented environment may contain active entities, static and dynamic objects, groups of entities. Some examples: "Each smart city starts with a number of households, vehicles and power stations. ... Its area is placed at the origin of a coordinated system or as a neighbor to an existing reference city e.g. on north, south, east or west" [31]. "The electricity market environment typically consists of a pool that players submit their bids to, which can be symmetric or asymmetric, and a floor for bilateral 
contracts" [30]. "Ten thousand agents that fill a corridor that is 300m long" [22]. "The floor plan contains a number of office spaces organized along hallways and corridors. There are two egress exits, exit A on the west and exit B on the south" [33]. "The static structure of a bus network is composed of four elements: itinerary, line, bus stop and bus station" [20]. "is a warehouse consisting of: number of Gates where the articulated lorries leave their containers waiting to be unloaded; number of Sorting Area with twelve Sorter Places (where the pallets are left in order to be addressed toward next destination)..." [29].

5) The problem statement describes the dynamics occurring in the real world portion. These dynamics are expressed in terms of actions and interactions of active entities. They can also be constrained by some regulations. To give some examples: "When people are crossing portals, care must be taken to avoid intersection between agents leaving and agents entering"[23]."High truck A is stopped in the lane closest to the sidewalk and obscures the pedestrian's view. Vehicle $B$ approaches the crosswalk from the second lane and the view of the driver is obscured too"[21]. "Players negotiating on the pool must prepare a bid for the 24 periods of the spot market ... The market operator must assure that the economical dispatch accounts for the specified conditions, which might imply removing entities that have presented competitive bids but whose complex conditions were not satisfied" [30], "a network of facilities is responsible for orchestrating all operations from the forest to the customers, including the operations of many sawmills" "Once dried, bundles are disassembled to be planed, cut to length, sorted, and graded according to standard rules or customer specifications" [34]. "In a normal situation(non-panic), people will respect lines and wait for others to walk first" [23]. "People movement can also be restricted due to environmental constraints imposed by the spatial geometries" [33]. "The length of time that elapses during an assembly depends on a specified manufacturing delay parameter. It also depends on whether the assembly is performed in parallel or sequentially" [28].

6) The problem statement may contain information about entity groupings and roles of the entities. Examples: "Members in a hierarchically structured group (such as families) tend to stay together and follow the leader"[33]. "Each household belongs to one city and it is randomly placed within the city area. Households have a number of appliances that consume and/or produce energy" [31].

7) The problem statement defines the objectives of the simulation study. Examples taken from the examined papers are: "Egress Analysis for building design"[33]. "Traffic analysis for support decision for example road safety" [21]. "Efficient management of electricity network"[31]. "The goal of both simulations was to service
TABLE I

KEY ELEMENTS AND THEIR MAPPING WITH THE ANALYZED DOMAINS.

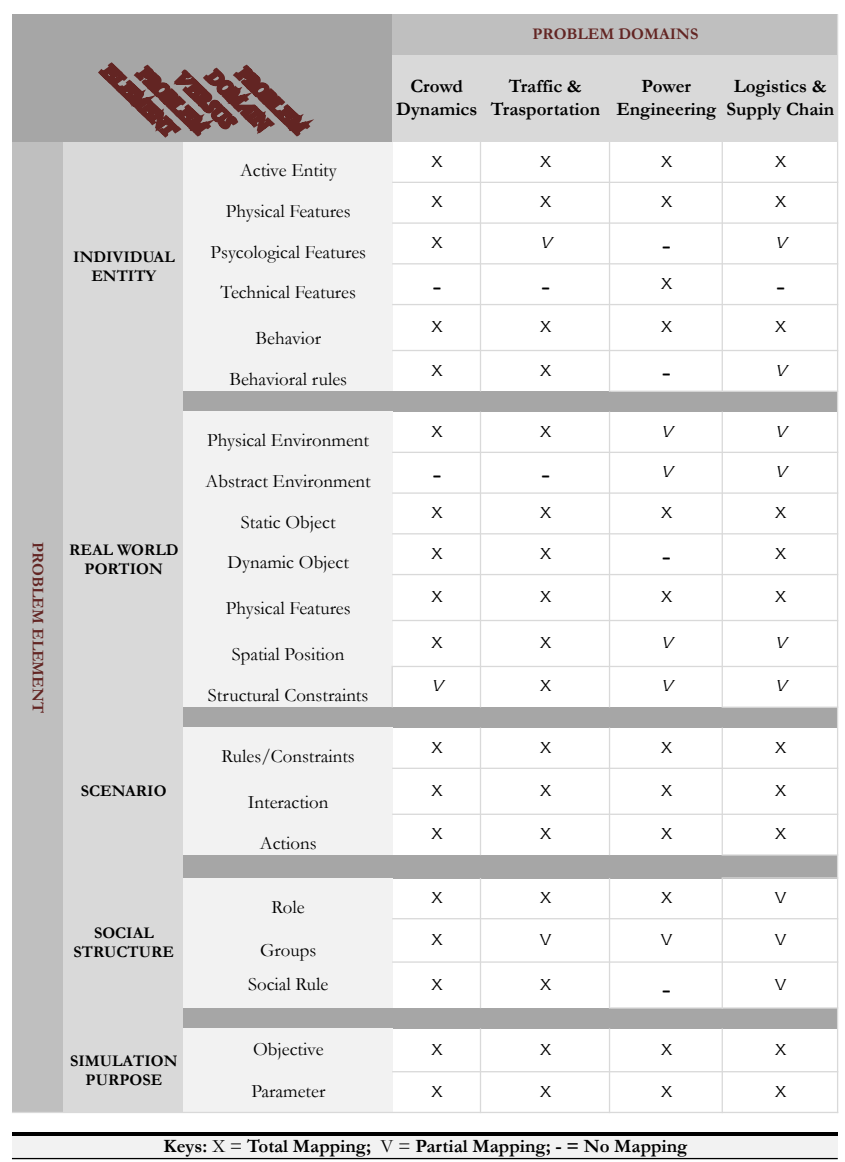

customer orders at or near 100\%, based on the three day requirement" [28].

8) The problem statement defines parameters to be tuned in order to reach simulation objectives. These parameters may refer to the system as a whole or to its entities. Some examples of system parameters: different spatial distribution of the occupant [33], density of crowd [22], changing supplier or add a distribution center [35]. Examples of entity parameters are: distance to obstacles[23], vehicles velocity and distance from cross walk [21], inventory levels, lead time and transportation time at different locations to better understand the dynamics of the supply chain [35].

After Key Concepts Identification, the Categorization of Concepts is carried out in order to find high level concepts as explained in Fig. 2. Two work products result from this activity, one describing the concepts (see Table I) and one describing the relationships discovered into the analyzed domain(see Table II).

In Table I we represent the set of high-level concepts resulting from this activity grouped according to a more general category they are related to. In particular, a total 
TABLE II

AN EXCERPT OF RELATIONSHIPS DETECTED AMONG CONCEPTS

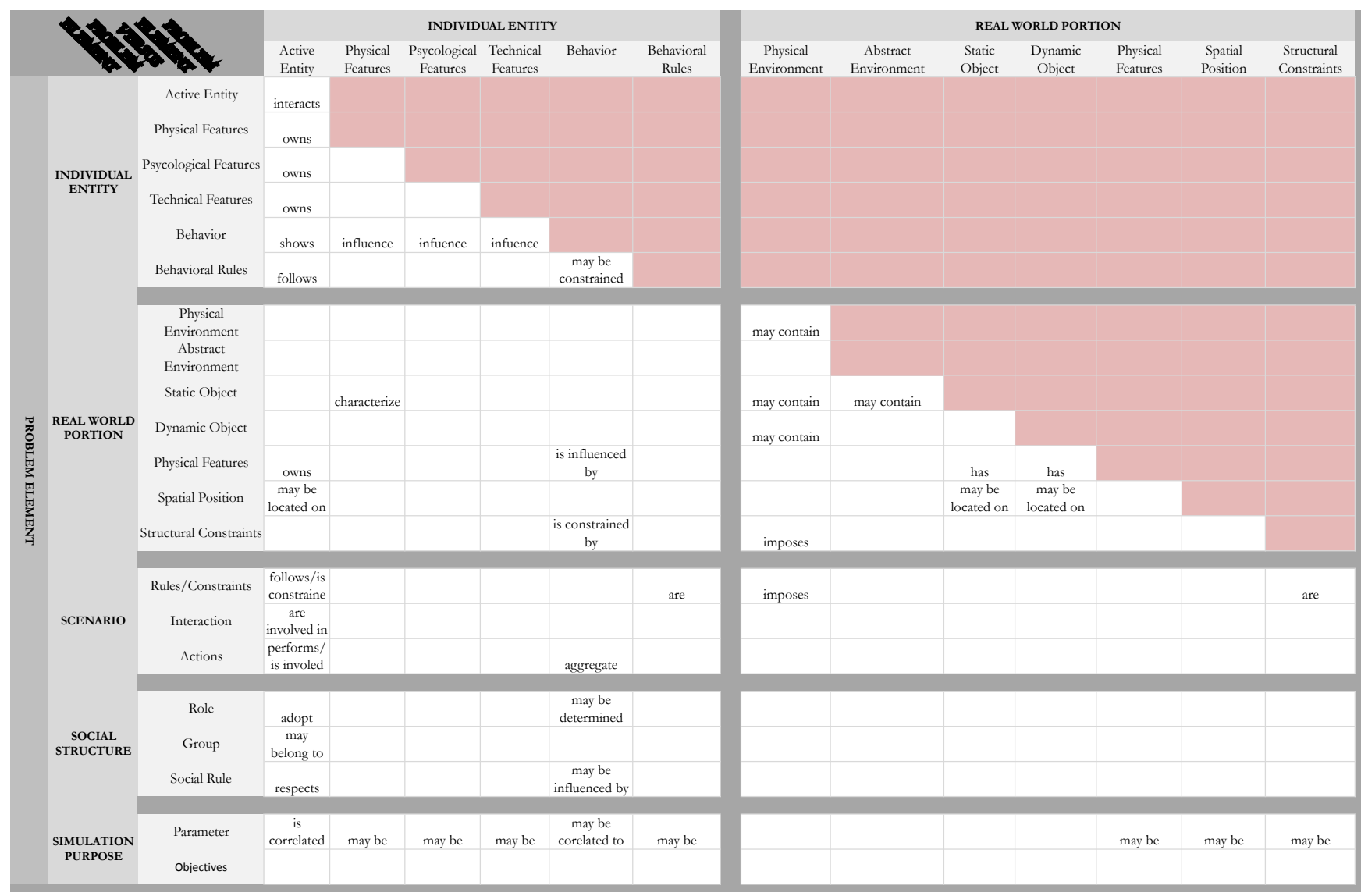

mapping ( $\mathrm{x}$ ) means that the concept has been encountered in all the papers we examined for the specific domain. Partial mapping (v) means that only some papers of the same domain use the concept the cell refers to. No mapping is represented by (-).

For space constraints, we only show an excerpt of the work product related to the relationships (see Table II). This is a table where the kind of relation discovered between two concepts is reported. The values of darker cells are the same of their symmetric cells. Only to name a few, we have detected the following relationships: $(i)$ an active entity interacts with active entities; (ii) the behavior may be constrained by behavioral rules; (iii) physical environment may contain static object; (iv) a static object may be located on a physical space and many others.

c) Metamodel Definition: The study of the simulation problems under analysis, Table I and Table II highlighted that, although the problem domains are quite different, they share some high level features and differ for some others. For instance, looking at the table's columns, we may see that Abstract Environment is not present in the Crowd Dynamics and Traffic and Transportation domains and it is only partially present in the other two. The Physical Environment has always been found in the Crowd Dynamics and Traffic and Transportation domains and only partially in the Power Engineering and in the Logistics and Supply Chain management domains. So, we may say that the problem statement metamodel for the simulation study of a specific domain may be composed of a core metamodel, where common elements for all the studied domain may be found, and extensions where some specific and particular elements are shown (Fig. 3).

\begin{tabular}{|c|c|c|}
\hline \multicolumn{3}{|c|}{ Core Metamodel } \\
\hline $\begin{array}{c}\text { Domain 1 } \\
\text { Extension } \\
\text { Content }\end{array}$ & $\begin{array}{c}\text { Domain 2 } \\
\text { Extension } \\
\text { Content }\end{array}$ & $\begin{array}{c}\text { Domain N } \\
\text { Extension } \\
\text { Content }\end{array}$ \\
\hline
\end{tabular}

Fig. 3. Metamodel Scheme

Hence, the Core Metamodel contains all the elements we identified derived from the set of concepts that are shared among all domains under analysis. As a consequence, the role of the Core Metamodel will be to provide a minimum set of elements to be described in a problem statement for a non specific agent based simulation study. The Domain Extension Contents are collections of metamodel elements that allow for defining "more specific and domain dependent" contents for a 
problem statement of agent based simulation studies belonging to a particular domain.

The resulting Core Metamodel is shown in Fig. 4. It aims to support the definition of an agent based simulation problem by identifying those elements that, once defined in the problem statement, will provide a shared starting point for the development of an agent based simulation model. The Domain Extension Contents for Traffic and Transportation and Power Engineering domains are shown in Fig. 5 and Fig. 6 respectively. As concerns, the Domain Extension Content for Crowds Dynamics domain, it entirely overlaps with that Traffic and Transportation Extension Content. For space constraints, we do not show the Logistics and Supply Chain Extension Content.

In particular, Table I, along with other considerations arisen from the activities we performed, helped us to define the elements of the metamodel. Whilst, Table II helped us to define the relationships among elements of the metamodel. The total or partial mapping also provided us information to define the cardinality of the metamodel relationships. For instance, from Table I we defined the metamodel elements: Active Entity, Behavioral Rule, Social Rule, Structural Constraint and Behavior. In Table II, we can see that these elements are linked by the following relationships: (i) Active Entity follows Behavioral Rules; (ii) Active Entity respects Social Rules; (iii) Behavior is constrained by Structural Constraints. Hence, we chose to model these relationships in our metamodel by introducing an abstract element named Rule whose Structural Constraints, Behavioral Rules and Social Rules are specializations. This choice allowed us to model the aforementioned relationships with only one link between Behavior and Rule labeled is constrained by. The Active Entity is related to Rule by means of its Behavior. In the same way, we deducted some other relationships and elements of the metamodel that do not explicitly appear in the tables.

In the following we describe the Core Metamodel elements showing also, when present, the related elements of the Domain Extension Contents.

Looking at Fig. 4, a generic Simulation Problem Statement is composed of a System to be simulated and Simulation Purposes. The System to be simulated in an agent based simulation study can be described by means of a set of interacting entities (i.e.: Active Entities) that own some distinctive attributes concerning their individuality (i.e.: Features) and that show particular Behaviors. A Physical Feature is a particular kind of Feature. It allows to describe the body properties of the entities (i.e: weight, height, etc...). The Feature may also be Psychological (i.e: impatient, polite, etc...) and Technical Feature (i.e.: energy consumption, capacity, etc...), we found the first in the description of the Crowd Dynamics domain and in the Traffic and Transportation domain (see the Extension Content in Fig. 5) whereas we found the second in the description of the Active Entities in the domain of Power Engineering (see the Extension Content in Fig. 6). Whilst, the Behavior is constrained by Rules. Rules can be Behavioral Rules (see Fig.5), Structural Constraints and Social Rules (see
Fig. 5). A Behavioral Rule is an explicit statement or principle governing the functionality, the conduct or the procedures within a particular domain, commonly prescribing what is possible or allowable. Social Rules and Structural Constraints are defined in the following.

Commonly, the primary purpose of a simulation study is to analyze the behavior of a system under some conditions. Thus, the simulation purpose is defined by identifying the Questions to be answered and by determining what are the Parameters to be investigated from which the resulting simulation model will depend on. In this context, a Parameter denotes a measurable factor that can be varied during simulation experiments and may characterize the system and/or determine its behavior (i.e: System Parameter). Parameters are related to each Active Entity present in the system so, since Active Entities own Features and show Behaviors, parameters may also characterize or determine Behavior and may be related to Features through the Entity Parameter. For instance the velocity of a vehicle is a physical feature described together with the related active entity, so, in this case, a physical feature may also be realized by an Entity Parameter.

Moreover, the System to be simulated concerns a Real-World Portion that includes Active Entities and passive entities (i.e.: Objects). These latter do not exhibit behaviors but contribute to the description of the System to be simulated. The RealWorld Portion is a Physical Environment in the domains of Crowd Dynamics and Traffic and Transportation and it may impose several Structural Constraints (see Fig. 5) derived by its physical configuration. Whilst, it can be also an Abstract Environment like it is in the domain of Power Engineering (see Fig. 6) for electricity markets.

In the same way, Objects may be Static or Dynamic Objects in the Crowd Dynamics and in the Traffic and Transportation domain but only static in the Power Engineering domain ${ }^{2}$ (see the Extension Contents in Fig. 5 and Fig. 6 respectively).

Moreover, Active Entities and Objects are located on a Spatial Position when the problem concerns a Physical Environment.

For describing the System to be simulated it is also necessary to identify Scenarios to be investigated. A Scenario is a description of the Interactions that occur among entities and/or the Actions individually performed by Active Entities. A Scenario describes a way in which the System to be simulated behaves in specific situations.

Finally, the System to be simulated may be further described by means of a Social Structure. The Social Structure describes the way the System to be simulated is organized according to specific Roles played by Active Entities. A Role describes what an active entity is able to do. It expresses a set of behaviors showed by an Active Entity when it is involved in a social

\footnotetext{
${ }^{2}$ We would like to point out again that these considerations are the result of a preliminary work. We think to be likely that Dynamic Objects can be found in the Power Engineering Domain, but at the moment this does not emerge from the examined papers. Whether the element Dynamic Objects should be present also in the domain of the Power Engineering, then both elements, Dynamic Object and Static Object, will be moved within the Core Metamodel.
} 


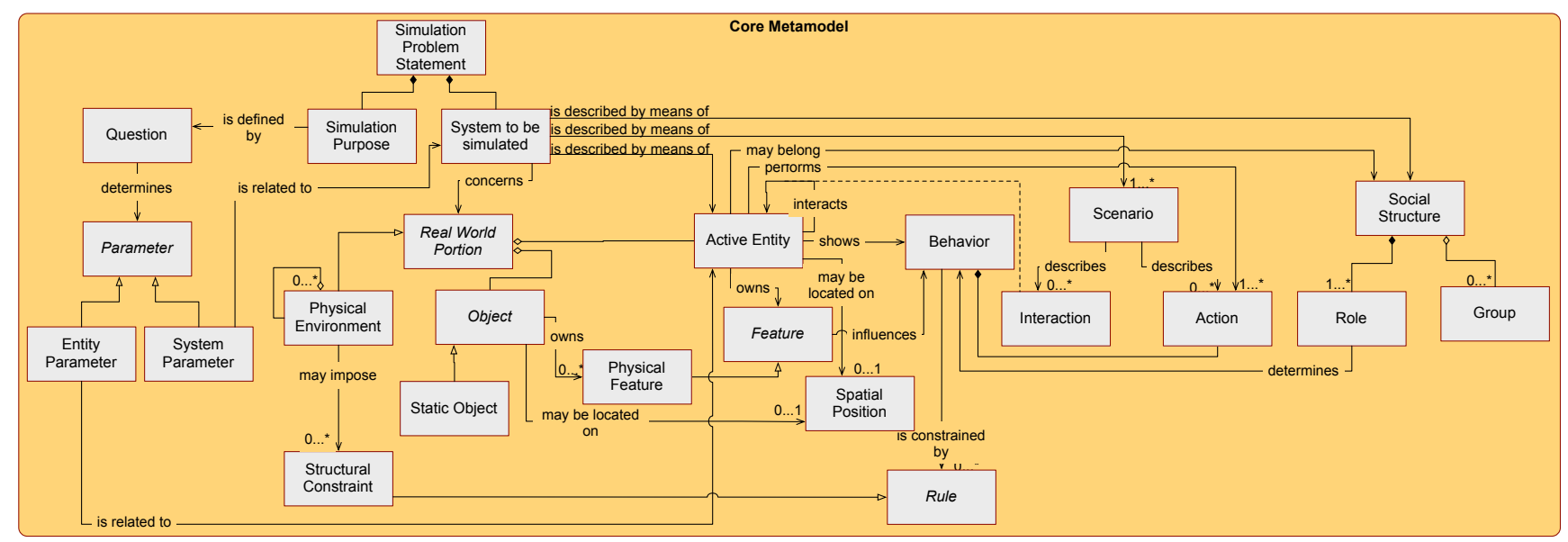

Fig. 4. The Core Metamodel derived from the domain under analysis.

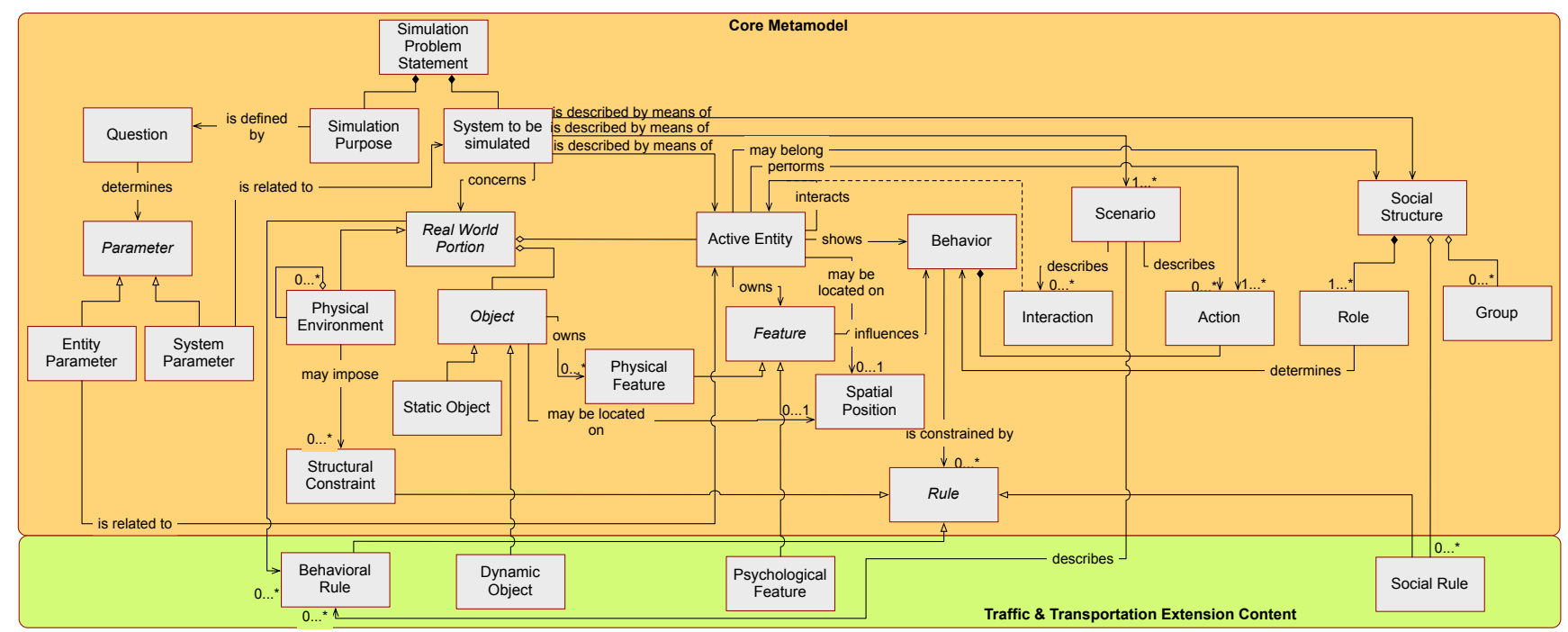

Fig. 5. Core Metamodel along with Traffic and Transportation Extension Content.

pattern. A Group defines an aggregate of active entities that can be together in the same place or that can be connected by some shared behavior or feature. A Social Rule has the same role of a Behavioral Rule but it is shared and followed by the members of a Group.

\section{Discussions}

The focus of the Problem Statement Activity is to acquire all the necessary knowledge of the real-world system to be simulated and to point out the issues that the simulation studies have to address. In particular, without a definitive statement of the specific questions of interest to be addressed, it is impossible to decide on an appropriate level of knowledge detail useful to build an appropriate simulation model. A method to clearly define what type of the knowledge about the problem is relevant may be useful in order to avoid missing important parts of the problem description. The metamodel resulting from our study allows to handle two levels of knowledge at the same time. A knowledge related to the system to be simulated as a whole. It concerns aspects of the system that are not directly related to the individual entities and it helps to build the socalled environment model ${ }^{3}$. A knowledge relating to specific aspects of single entities that will be suited for building the agent models. Moreover, preliminary results we have obtained give us some suggestions about the knowledge to be included in a problem statement and how to describe it in a systematic way. The Core Metamodel highlights that the formulation of a simulation study may ground on five key aspects:

\footnotetext{
${ }^{3}$ An agent based simulation model is in many cases composed of two basic components. They are an agent model that focuses on each single agent and its behavior and an environment model that focuses on the structure and features of the real world portion in which agents act.
} 


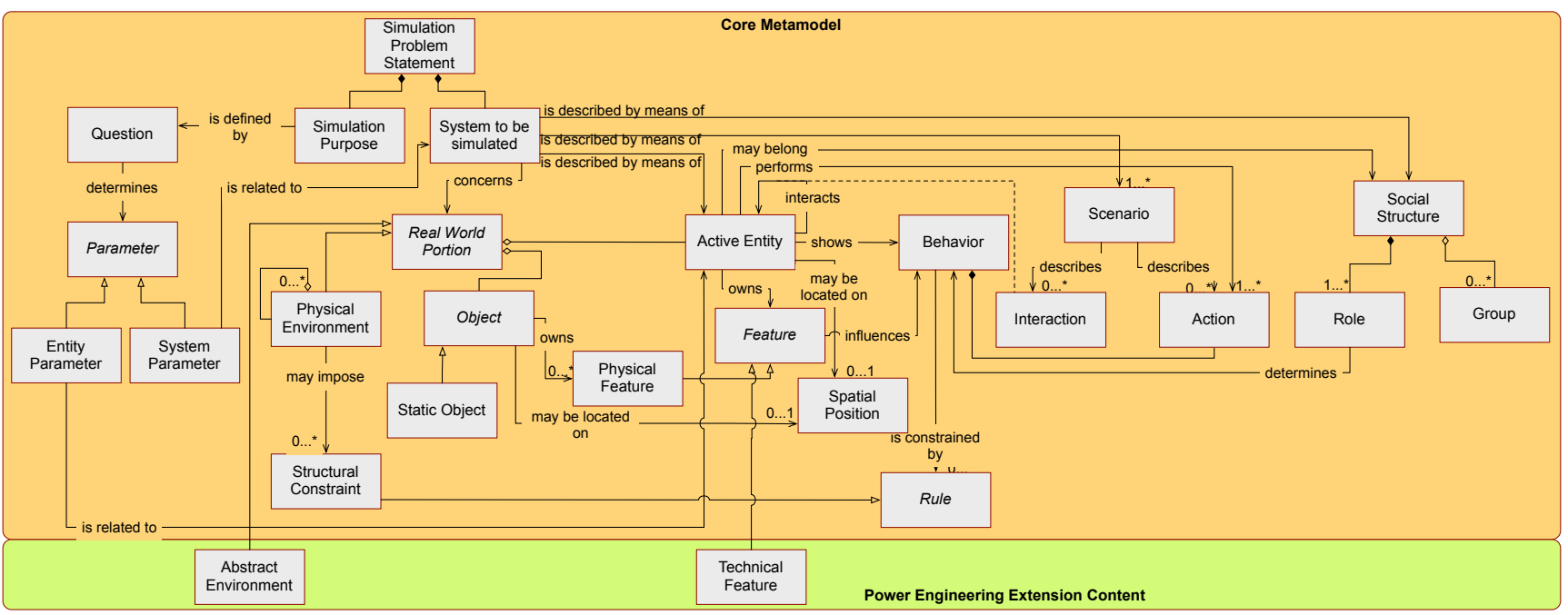

Fig. 6. Core Metamodel along with Power Engineering Extension Content.

1) the issues to be addressed by the simulation study made explicit by means of the metamodel element named Simulation Purpose. Instantiating this metamodel element means to formulate the questions to be answered that coincide with the definition of goals of the simulation study. As a consequence, this leads to associate goals with information that define them operationally and that has to be considered during the definition of the simulation model.

2) the structural aspect of the system to be simulated defined by its constituting parts. It is made explicit by instantiating the metamodel element named Real World Portion. This means to collect the knowledge necessary to describe the real-world part involved in the system to be simulated according to the issues to be addressed. Commonly, this concerns the description of the physical configuration of the real-world system under analysis (i.e: its components). As the Core metamodel shows, components may be static objects of the portion of the world enclosed by the system or active entities that are someone/something performing actions;

3) the dynamical aspect of the system to be simulated caused by processes taking place in the system. It is captured by defining actions and interactions that are performed by someone/something in particular operative scenarios.

4) the organizational aspect (if any) which refers to the social structure captured by defining the groups and sets of roles that may be present in the system to be simulated.

5) the normative aspect (if any) given by regulations that commonly constrains the dynamics or physicality of the system. It refers to any kind of regulations such as procedural rules, legal regulations, social norms, structural rules or physical laws. Usually, only some kinds of norm affect the system, this strongly depends on the particular domain. For example, in a simulation study concerning a manufacturing system normally the system is subjected to procedural rules and legal regulations. Vice versa, if the simulation is about the citizen of a town during an event then, probably, social norms could affect the system.

These key aspects may provide a first guideline about the kind of knowledge that can be managed during the problem statement activity. Moreover, basing on such a metamodel we are able to establish a starting point for the construction of a systematic approach for performing the problem statement activity by choosing the order that should be used for instantiating the metamodel elements. We already explored such an approach with the PRoDe method for developing software design processes starting from the underlying metamodel [14].

\section{CONCLUSIONS AND REMARKS}

Multiagent simulations are employed in several domains and for several simulation purposes. Finding a uniform way to address the problem description for a simulation study is an open issue. The work proposed in this paper is a preliminary step toward an ambitious objective: the definition of a complete methodological approach to obtain high quality simulation models for generic agent-based simulation studies. Such an approach will cover several activities starting from the problem statement to the results analysis trough the model validation. At the moment, we are addressing some questions related to the problem statement activity and in this paper we present our preliminary results.

As well as in many engineering contexts, we think that an accurate problem formulation is the first step to be accomplished in order to obtain a high-quality agent based simulation model. To do this, we are defining a metamodelbased approach in order to perform the problem statement 
activity. The metamodel aims to guide the problem formulation highlighting the main elements and relationships that have to be made explicit. In this paper we present the rough metamodel we have already defined. This metamodel is composed of elements belonging to a shared level among several simulation problem domains useful for formulating generic simulation studies and several domain extension contents that allow us for detailing specific domain simulation studies.

\section{REFERENCES}

[1] F. Klügl, "Engineering agent-based simulation models?" in AgentOriented Software Engineering XIII, ser. Lecture Notes in Computer Science, J. P. Müller and M. Cossentino, Eds. Springer Berlin Heidelberg, 2013, vol. 7852, pp. 179-196. ISBN 978-3-642-39865-0

[2] O. Labarthe, E. Tranvouez, A. Ferrarini, B. Espinasse, and B. Montreuil, "A heterogeneous multi-agent modelling for distributed simulation of supply chains," in Holonic and Multi-Agent Systems for Manufacturing, ser. Lecture Notes in Computer Science, V. Marík, D. McFarlane, and P. Valckenaers, Eds. Springer Berlin Heidelberg, 2003, vol. 2744, pp. 134-145. ISBN 978-3-540-40751-5

[3] O. Balci, "Guidelines for successful simulation studies," in Simulation Conference, 1990. Proceedings., Winter, Dec 1990. doi 10.1109/WSC.1990.129482 pp. 25-32.

[4] R. E. Nance, "The conical methodology and the evolution of simulation model development," Annals of Operations Research, vol. 53, no. 1, pp. 1-45, 1994. doi: $10.1007 / \mathrm{BF} 02136825$

[5] A. Law, "How to build valid and credible simulation models," in Simulation Conference (WSC), Proceedings of the 2009 Winter, Dec 2009. doi: $10.1109 /$ WSC. 2009.5429312 pp. 24-33.

[6] C. M. Macal and M. J. North, "Tutorial on agent-based modeling and simulation," in Proceedings of the 37th conference on Winter simulation. Winter Simulation Conference, 2005. doi: 10.1057/jos.2010.3 pp. 2-15.

[7] O. Balci and R. E. Nance, "Formulated problem verification as an explicit requirement of model credibility," SIMULATION, vol. 45 , no. 2 , pp. 76-86, 1985. doi: 10.1177/003754978504500204

[8] A. Garro and W. Russo, " $<\mathrm{i}>$ easyabms $</ \mathrm{i}>$ : A domain-expert oriented methodology for agent-based modeling and simulation," Simulation Modelling Practice and Theory, vol. 18, no. 10, pp. 1453-1467, 2010.

[9] S. Viller and I. Sommerville, "Ethnographically informed analysis for software engineers," International Journal of Human Computer Studies, vol. 53, no. 1, pp. 169-196, 2000. doi: 10.1006/ijhc.2000.0370

[10] M. Cossentino and V. Seidita, "PASSI: Process for agent societies specification and implementation," in Handbook on Agent-Oriented Design Processes, M. Cossentino, V. Hilaire, A. Molesini, and V. Seidita, Eds. Springer Berlin Heidelberg, 2014, pp. 287-329. ISBN 978-3-64239974-9

[11] A. Chella, M. Cossentino, L. Sabatucci, and V. Seidita, "Agile passi: An agile process for designing agents," Computer Systems Science and Engineering, vol. 21, no. 2, pp. 133-144, 2006.

[12] M. Cossentino, V. Hilaire, N. Gaud, S. Galland, and A. Koukam, "The ASPECS process," in Handbook on Agent-Oriented Design Processes, M. Cossentino, V. Hilaire, A. Molesini, and V. Seidita, Eds. Springer Berlin Heidelberg, 2014, pp. 65-114. ISBN 978-3-642-39974-9

[13] V. Seidita, M. Cossentino, and S. Gaglio, "Adapting PASSI to support a goal oriented approach: a situational method engineering experiment," in Proc. of the Fifth European workshop on Multi-Agent Systems (EUMAS'07), 2007.

[14] V. Seidita, M. Cossentino, V. Hilaire, N. Gaud, S. Galland, A. Koukam, and S. Gaglio, "The metamodel: a starting point for design processes construction," International Journal of Software Engineering and Knowledge Engineering, vol. 20, no. 04, pp. 575-608, 2010. doi: $10.1142 / \mathrm{S} 0218194010004785$

[15] K. Boer-Sorban, Agent-Based Simulation of Financial Markets: A Modular, Continuous-time Approach. Erasmus Research Institute of Management (ERIM), 2008, no. EPS-2008-119-LIS

[16] M. Lovric, Behavioral Finance and Agent-Based Artificial Markets. Erasmus Research Institute of Management (ERIM), 2011, no. EPS2011-229-F\&A.
[17] I. Benenson, "Multi-agent simulations of residential dynamics in the city," Computers, Environment and Urban Systems, vol. 22, no. 1, pp. 25-42, 1998. doi: 10.1016/S0198-9715(98)00017-9

[18] J. Perret, F. Curie, J. Gaffuri, and A. Ruas, "A multi-agent system for the simulation of urban dynamics," in 10th European Conference on Complex Systems (ECCS'10), Lisbon, Portugal, 2010.

[19] P. Paruchuri, A. R. Pullalarevu, and K. Karlapalem, "Multi agent simulation of unorganized traffic," in Proceedings of the first international joint conference on Autonomous agents and multiagent systems: part 1 . ACM, 2002. doi: $10.1145 / 544741.544786$ pp. 176-183.

[20] D. Meignan, O. Simonin, and A. Koukam, "Simulation and evaluation of urban bus-networks using a multiagent approach," Simulation Modelling Practice and Theory, vol. 15, no. 6, pp. 659-671, 2007. doi: 10.1016/j.simpat.2007.02.005

[21] G. Waizman, S. Shoval, and I. Benenson, "Micro-simulation model for assessing the risk of car-pedestrian road accidents," in 7th International Workshop on Agents in Traffic and Transportation, Valencia, Spain, 2012

[22] S. J. Guy, J. Chhugani, S. Curtis, P. Dubey, M. Lin, and D. Manocha, "Pledestrians: a least-effort approach to crowd simulation," in Proceedings of the 2010 ACM SIGGRAPH/Eurographics Symposium on Computer Animation. Eurographics Association, 2010, pp. 119-128.

[23] N. Pelechano, J. M. Allbeck, and N. I. Badler, "Controlling individual agents in high-density crowd simulation," in Proceedings of the 2007 ACM SIGGRAPH/Eurographics symposium on Computer animation. Eurographics Association, 2007, pp. 99-108.

[24] A. Shendarkar, K. Vasudevan, S. Lee, and Y.-J. Son, "Crowd simulation for emergency response using bdi agent based on virtual reality," in Proceedings of the 38th conference on Winter simulation. Winter Simulation Conference, 2006. doi: 10.1109/WSC.2006.323128 pp. 545553.

[25] F. Menges, B. Mishra, and G. Narzisi, "Modeling and simulation of e-mail social networks: a new stochastic agent-based approach," in Proceedings of the 40th Conference on Winter Simulation. Winter Simulation Conference, 2008, pp. 2792-2800.

[26] P. Holme and G. Ghoshal, "Dynamics of networking agents competing for high centrality and low degree," Physical review letters, vol. 96, no. 9, p. 098701, 2006. doi: 10.1103/PhysRevLett.96.09870

[27] L. A. de Santa-Eulalia, J.-M. Frayret, and S. D'Amours, "Essay on conceptual modeling, analysis and illustration of agent-based simulations for distributed supply chain planning," INFOR, vol. 46, no. 2, pp. 97116, 2008.

[28] S. Buckley and C. An, "Supply chain simulation," in Supply Chain Management on Demand, C. An and H. Fromm, Eds. Springer Berlin Heidelberg, 2005, pp. 17-35. ISBN 978-3-540-24423-3

[29] M. Cossentino, C. Lodato, S. Lopes, and P. Ribino, "Multi agent simulation for decision making in warehouse management," 2011 Federated Conference on Computer Science and Information Systems, FedCSIS 2011, pp. 611-618, 2011

[30] Z. Vale, T. Pinto, I. Praça, and H. Morais, "Mascem: electricity markets simulation with strategic agents," Intelligent Systems, IEEE, vol. 26, no. 2, pp. 9-17, 2011. doi: 10.1109/MIS.2011.3

[31] S. Karnouskos and T. N. De Holanda, "Simulation of a smart grid city with software agents," in Computer Modeling and Simulation, 2009 EMS'09. Third UKSim European Symposium on. IEEE, 2009. doi: 10.1109/EMS.2009.53 pp. 424-429.

[32] N. W. Oo and V. Miranda, "Multi-energy retail market simulation with intelligent agents," in Power Tech, 2005 IEEE Russia. IEEE, 2005. doi 10.1109/PTC.2005.4524755 pp. 1-7.

[33] X. Pan, C. S. Han, K. Dauber, and K. H. Law, "A multi-agent based framework for the simulation of human and social behaviors during emergency evacuations," Ai \& Society, vol. 22, no. 2, pp. 113-132, 2007. doi: 10.1007/s00146-007-0126-1

[34] J.-M. Frayret, S. D'amours, A. Rousseau, S. Harvey, and J. Gaudreault, "Agent-based supply-chain planning in the forest products industry," International Journal of Flexible Manufacturing Systems, vol. 19, no. 4, pp. 358-391, 2007. doi: $10.1007 / \mathrm{s} 10696-008-9034-z$

[35] J. M. Swaminathan, S. F. Smith, and N. M. Sadeh, "Modeling supply chain dynamics: A multiagent approach," Decision Sciences, vol. 29 no. 3, pp. 607-632, 1998. doi: 10.1111/j.1540-5915.1998.tb01356.x 